beschneidet er die eigentümliche Radikalität jener Proteste um ihre subjektive Dimension. Denn den Revolten der späten sechziger Jahre lagen in erster Linie keine materiellen Interessen zugrunde; sie verdankten sich vor allem einer breiten postmaterialistischen Strömung, die zur selben Zeit die Jugend in allen Industriegesellschaften erfasst hatte.

Eine zweite nicht weniger folgenreiche Beschneidung macht sich hier bemerkbar: Greven glaubt, sich in seiner Untersuchung auf die Bundesrepublik beschränken zu können. Durch diese Einschränkung verschwinden soziale Konstitutionsbedingungen aus dem Blickfeld, die für die Entstehung von Protestbewegungen in den verschiedensten Erdteilen ausschlaggebend waren. Zudem wird ein so entscheidender politischer Konstitutionsfaktor wie der Ost-West-Konflikt einfach ausgeblendet. Die Frage etwa, warum die bundesdeutsche Version der 68er-Bewegung (ebenso wie kurze Zeit später die terroristische RAF) in West-Berlin entstanden ist, wird überhaupt nicht gestellt.

Ein anderes wesentliches Problem besteht darin, dass hier keine überzeugende historische Interpunktion vorgenommen wird. Weil Greven einen konventionellen Begriff des Politischen favorisiert, neigt er dazu, den Beginn der außerparlamentarischen Bewegung immer weiter zurückzuverlagern. Bei dieser Suche nach den Ursprüngen landet er schließlich - ohne dafür irgendein Kriterium zu benennen - in den fünfziger Jahren. Dabei wäre es unter der Voraussetzung des von ihm ins Zentrum gestellten Begriffs der Systemopposition eigentlich ganz einfach, eine historisch plausible Einordnung vorzunehmen; denn die APO war im Kern eine Parallelerscheinung zur ersten Großen Koalition. Diese dauerte vom Dezember 1966 bis zum Oktober 1969. Und genau das war der Zeitraum, in dem jene Protestbewegung von sich reden machte, die heute gewiss verkürzend als die der 68er bezeichnet wird. Weil es keine überzeugende innerparlamentarische Opposition mehr gab, kam der Anstoß zu längst überfälligen Reformen ebenso wie zu revolutionär deklarierten Vorhaben von außen.

Mit seiner Untersuchung des immer noch verwirrenden Phänomens der 68er-Bewegung hatte Greven in Aussicht gestellt, durch Verwendung des Begriffs Systemopposition ein höheres Maß an Rationalität zu erreichen. Herausgekommen ist dabei jedoch ein in vielfacher Hinsicht nicht unproblematisches Resultat. Gerade er, der einer Vielzahl von anderen Forschern vorwirft, sie bedienten sich eines erinnerungspolitischen Konstrukts, hat sich sein Untersuchungsfeld so konstruiert, dass er nichts anderes dabei herausgefunden, als er zuvor hineingesteckt hat.

Wolfgang Kraushaar

\title{
Amt und Person des Alterspräsidenten: das Für und Wider eines parlamentarischen Kleinods
}

Brunner, Benedikt: Der Alterspräsident. Ein Konstituierungsreglement und seine Alternativen, VS Verlag für Sozialwissenschaften, Wiesbaden 2012, 350 Seiten, € 49,95.

Dem Alterspräsidenten von Parlamenten wird in der Regel wenig Bedeutung beigemessen. Dementsprechend überschaubar ist der Forschungsstand. Außer der vorliegenden Promotionsschrift von Benedikt Brunner gibt es keine genuin politikwissenschaftliche Untersuchung, die sich dezidiert dieser traditionsreichen parlamentarischen Institution widmet. Dabei zeigt 
der Autor, dass diese „evolutionär betagte Eintagsfliege“ (S. 19) durchaus gewinnbringend untersucht werden kann. Obwohl Alterspräsidentschaften in der Regel „harmlos und unspektakulär" (S. 20) sind, rückt das Interesse und die Kritik an Amt und Person besonders dann ins Zentrum politischer und medialer Aufmerksamkeit, wenn absehbar ist, dass es Vertreter extremistischer Parteien ausüben werden. Prominente Beispiele sind die Alterspräsidentschaften des französischen Front National-Abgeordneten Claude Autant-Lara 1989 im Europäischen Parlament oder des parteilosen Stefan Heym, der über die offene Liste der PDS 1994 in den Bundestag eingezogen war. Zum Teil reagierten Parlamente zur Vermeidung von unliebsamen Alterspräsidenten mit einer Änderung des Konstituierungsverfahrens. Neben einer Parlamentseröffnung durch den ältesten Abgeordneten gibt es die Möglichkeit, die erste Sitzung einer Wahlperiode durch den dienstältesten Parlamentarier (Dienstaltersregelung) oder durch einen Vertreter des Präsidiums der vergangenen Wahlperiode (Präsidiumsregelung) eröffnen zu lassen. An dieser Stelle setzt das Erkenntnisinteresse von Brunner an: „Wie sinnvoll sind die einzelnen Handlungsoptionen, was spricht für und was spricht gegen sie?" (S. 22) Grundlage der detaillierten qualitativen Analyse sind zehn Parlamente in Deutschland, Österreich, der Schweiz und das Europäische Parlament, die nach zwei Kriterien ausgesucht wurden: Volksvertretungen, (1) die eine Änderung oder Abschaffung des Alterspräsidentenamtes vornahmen oder (2) in denen es tatsächliche oder potentielle Konflikte um die Person eines Alterspräsidenten gab.

Die gut lesbare Arbeit besticht durch ihre breite und tiefgehende Anlage. Nachdem zunächst die historischen Grundlagen des Amtes in der Französischen Revolution und die parlamentarischen Ursprünge der Alterspräsidentenreden in den ausgesuchten Volksvertretungen nachgezeichnet werden, untersucht Brunner die unterschiedlichen Amtsoptionen anhand der zehn Fallbeispiele. Besondere Aufmerksamkeit widmet er dabei den umstrittenen Alterspräsidentschaften und Reformdiskussionen. Auf dieser Grundlage klärt er sodann aufschlussreich rechtliche, politische und personelle Rahmenbedingungen. Im Anschluss lenkt er seinen Untersuchungsfokus auf die Reden derjenigen, die die erste Sitzung einer Wahlperiode eröffnen. Die vielfältigen Interpretationsergebnisse, die auf der Auswertung rechtlicher Quellen wie Verfassungen, Geschäftsordnungen oder Geschäftsordnungsgesetzen, zahlreicher Artikel lokaler und überregionaler Printmedien, stenographischer Berichte, Parlamentsdrucksachen, Parlamentshandbüchern, Parlamentswebsites sowie (auto-)biographischer Schriften beruhen, fasst er konzentriert und übersichtlich in seinem Schlussteil zusammen. Eckhard Jesse, der diese Promotion betreut und ein Vorwort zur Publikation beigesteuert hat, ist beizupflichten, wenn er schreibt, dass die „in Tabellenform gekleideten Ergebnisübersichten im Schlussteil [...] ein Musterbespiel an stringenter Systematik“ (S. 18) sind. Brunner stellt hier zum einen die Unterschiede und Gemeinsamkeiten der drei Amtsoptionen anhand der von ihm untersuchten 19 Merkmalsausprägungen dar (vgl. S. 334 ff.). ${ }^{1}$ Zum anderen listet er sieben Aspekte auf, die bei den Handlungsoptionen Beibehaltung der Lebensaltersregelung, ihrer Modifikation hin zu einer Dienstaltersregelung oder deren Abschaffung

1 Diese sind Geschichte und Verbreitung des Amtes und der Rede, Vertretungsregelung außerhalb der Eröffnungssitzung, Parlamentsautonomie, Diskontinuitätsgrundsatz, Maßnahmenspektrum während der Konstituierung, negative wie positive Planbarkeit der Amtsinhaber, Partei- beziehungsweise Fraktionszugehörigkeit, Begleitumstände der Rede, sechs Redefunktionen, Themensetzung, Polarisierungsgrad der Reden. 
zugunsten einer Präsidiumsregelung als Ergebnis seiner Untersuchung zu bedenken sind (vgl. S. 341 f.). ${ }^{2}$

Besonders eindrücklich mahnt Brunner an, Änderungen des Konstituierungsreglements nicht ad hoc und anlassbezogen durchzuführen. Neben dem „Risiko, überstürzt und unreflektiert zu handeln“, bleibe der „Geschmack eines ,faulen Tricks“" (S. 234). Zudem sei es fraglich, „ob die demokratischen Kräfte in einem Parlament den extremistischen oder radikalen Strömungen einen solchen zwar indirekten, aber spürbaren Einfluss auf ihre Handlungen gewähren sollten“ (S. 343). Darüber hinaus könnten jene einen symbolträchtigen Sieg davontragen, wenn sie entgegen ihrem bisherigen Verhalten gegen Änderungen klagten. Besonders bei den häufig in den Geschäftsordnungen verankerten Regelungen bestünden rechtliche Unklarheiten, ob sie durch eine einfache Geschäftsordnungsänderung oder eher durch eine Verfassungsänderung herbeizuführen seien.

Obwohl Brunner beständig selbstkritisch anmerkt, dass seine qualitativen Befunde nur begrenzt verallgemeinerbar sind und keine Prognosen zulassen, kann er zeigen, dass selbst Vertreter extremistischer Parteien „sich dazu genötigt sehen, vom Stuhl des Alterspräsidenten aus moderate, ja staatstragende Reden zu halten“ (S. 345). Der häufig bemühte Anlass, eine über zweihundertjährige Tradition über Bord zu werfen, erweist sich somit in den untersuchten gefestigten Demokratien als weniger gefährlich als allgemein wahrgenommen. Dies spricht jedoch nicht gegen die Möglichkeit, wohlüberlegt Änderungen vorzunehmen, da eben auch die anderen zwei Amtsoptionen ihre Vorzüge haben. Es gibt hier „keine eindeutig richtige oder falsche Wahl“" (S. 343).

Die vorliegende Studie, die eine Diskussionsgrundlage schaffen möchte, falls „erneut in einer deutschen oder europäischen Volksvertretung das Amt des Alterspräsidenten aufgrund einer umstrittenen Person infrage gestellt wird" (S. 344), bietet eine umfassende Bestandsaufnahme von Pro- und Kontra-Argumenten für alle drei Amtsoptionen. Brunners Schrift kann daher im Falle künftiger Änderungsabsichten aufmerksame Leser unter Abgeordneten und Mitarbeitern der Parlamentsverwaltungen finden und helfen, die Optionen sorgfältig zu eruieren. Darüber hinaus dürfte die Untersuchung dieses parlamentarischen Kleinods allen an Parlamentskultur Interessierten eine vergnügliche und gewinnbringende Lektüre sein.

Marcus Wittig

2 Es sind der rechtlicher Rahmen des Amtes und der Rede, Anlass- und Ad-hoc-Gesetzgebung, die Stärkung randständiger Kräfte, Mehrheitsverhältnisse und Wegfall der Redefunktion. 